\title{
Dengue: Recent Trends
}

\author{
Rabindran ${ }^{1}$, Gedam DS ${ }^{2}$
}

Dr. Rabindran, Consultant Neonatologist, Billroth Hospital, Chennai, Dr D Sharad Gedam, Professor of Pediatrics L N Medical College, Bhopal, MP, India.

Address for correspondence: Dr Rabindran, E mail: rabindranindia@yahoo.co.in

\begin{abstract}
Today dengue ranks as the most important mosquito-borne viral disease in the world \& its incidence has increased 30 fold over the last 50 years. In India there is recent increase in cases of Dengue in last few years.
\end{abstract}

Keywords: Dengue, Recent trends, Dengue hemorrhagic fever

Dengue, an acute viral disease described in1780 by Benjamin Rush as "break bone fever" is caused by any of 4virus serotypes (DEN 1-4) \& is characterized by sudden onset of fever, headache, myalgia, rash, nausea, $\&$ vomiting. Severe forms of this disease are dengue hemorrhagic fever (DHF) characterized by petechiae, purpura, mild gum bleeding, nosebleeds, menorrhagia, or gastrointestinal bleeding \&dengue shock syndrome (DSS).Today dengue ranks as the most important mosquito-borne viral disease in the world \& its incidence has increased 30 -fold over the last 50 years. Upto 50 million infections occur annually with 500000 cases of DHF and 22,000 deaths mainly among children [1].Treated DHF/DSS is associated with a $1 \%$ mortality rate while mortality rate among untreated cases escalates to $20 \%$.The disease causes an estimated annual loss of 750000 disability-adjusted-life years [2]. Prior to 1970, only 9 countries experienced Dengue epidemics; however, the disease is now endemic in more than 120 countries in Africa, America, the Eastern Mediterranean, South-east Asia \& the Western Pacific.

WHO 2020 targets for dengue is to reduce morbidity by $25 \%$ \& mortality by $50 \%$.Determinant factors of dengue global epidemiology trends include 1) demographic changes including population growth, economical trends in tropical countries \& land use patterns; 2) increased urban population size \& density due to rural to urban migration 3) modern transportation with increased movement of people, commodities, animals, vectors \& pathogens; 4) changes in public health policies \& infrastructures [3]. The geographic spread of both the mosquito vectors \& the viruses has led to a global resurgence of epidemic dengue disease.Particularly, the disease is growing most rapidly in tropical \& subtropical countries where majority of the world's population resides thereby increasing health \&economic burden[4]. The only comforting news is that reported case-fatality rates have been lower in recent years than in the decades before 2000 .

The Global Strategy for Dengue Fever/ DHF Prevention and Control is more than ten years old but remains essentially unchanged. It comprises 5 major elements: selective integrated vector control, with community \&intersectoral participation; active disease surveillance based on a strong health-information system; emergency preparedness, capacity building \& training; $\&$ vector-control research. Since the last Scientific Working Group (2000), several new strategies have been developed like Rapid commercial diagnostic tests, Audiovisual guide, Global strategic framework for 
integrated vector management, Dengue CD-ROM (Wellcome Trust), Entomological survey technique, Sequencing of the Aedes. aegyptigenome \&DengueNet for global dengue surveillance. Seven insecticide products are available for mosquito larviciding ( 5 insect growth regulators, 2 bacterial larvicides), four approved for use in drinking-water (methoprene EC, pyriproxyfen GR, Vectobac DT \& GR); three for space spray applications to control mosquitoes (all pyrethroids).

New initiatives include Paediatric Dengue Vaccine Initiative (Bill \& Melinda Gates Foundation), Innovative Vector Control Consortium (Bill \& Melinda Gates Foundation), Asia-Pacific Dengue Partnership, DENFRAME \&DENCO (European Union), Involvement of Regional Development Banks (Asian Development Bank, Inter-American Development Bank) \&Streams of dengue research \& training supported by the UNICEF-UNDP-World Bank. Systems like Google Dengue Trends complements the current surveillance system, as it is both rapid \& costeffective.Geographic information systems (GIS) can visualize the spatiotemporal pattern and variation in disease risk $\&$ are widely useful in vector borne disease epidemiology [5].A Phase 1b study has been conducted to evaluate the immunogenicity \&safety of two dengue vaccine candidates[6].Chimaeric dengue vaccines have been developed combining the DNA coding for the envelope antigens of the dengue serotype with the 17D yellow fever vaccine to produce a replicating viral particle manifesting the dengue serotype envelope antigens with the core of the YF vaccine. In this format, ChimeriVax-DEN2 has been demonstrated to produce neutralizing antibodies to all four dengue serotypes in association with yellow fever vaccine pre-immunity [7].
In summary, the global trends of dengue disease epidemiology are characterized by a rapidly expanding geographic distribution of vector infestation, the risk of infection \&disease transmission, despite ongoing control efforts. Surveillance \& development of licensed vaccines on an urgent basis is mandatory to prevent emergence of dengue epidemics.

\section{Reference}

1. Chakravarti A, Kumaria R. Eco-epidemiological analysis of dengue infection during an outbreak of dengue fever, India. Virol J 2005;2:32.

2. Singhasivanon P, Jacobson J. Foreward. J ClinVirol2009; 46 (Sup 2): S1- S2.

3.Ginsberg J, Mohebbi MH, Patel RS, Brammer L, Smolinski MS, Brilliant L. Detecting influenze epidemics using search engine query data. Nature 2008;457:1012-1014.

4. Toledo ME, Rodrigues A, Valdes L, Carrion R, Cabrera G, Banderas D, et al. Evidence on impact of community-based environmental management on dengue transmission in Santiago deCuba. Trop Med Int Health 2011; 16: 744-747.

5. Robertson C, Nelson TA (2010) Review of software for space-time disease surveillance. Int J Health Geogr 9: 9-16.

6. Kitchener S, Nissen M, Nasveld P, Forrat R, Yoksan S, Lang J, Saluzzo JF. Immunogenicity and safety of two live-attenuated tetravalent dengue vaccine formulations in healthy Australian adults. Vaccine 2006; 24(9):1238-1241.

7. Guirakhoo F, Kitchener S, Morrison D, etal. Live attenuated chimeric yellow fever dengue type 2 (ChimerVax- DEN2) vaccine: phase I clinical trial for safety and immunogenicity in induction of cross neutralizing antibody responses to all 4 dengue serotypes. Hum Vaccin 2006; 2: 60-67.

\section{How to cite this article?}

Rabindran, Gedam DS. Dengue: Recent Trends. Int J Med Res Rev 2015;3(8):781-782. doi: 10.17511/ijmrr.2015.i8.169. 